\title{
Designing a Gamma Hydroxybutyrate (GHB) Structured Telephone-Administered Survey Instrument
}

\author{
Jo E. Dyer, PharmDa,b, Ilene B. Anderson, PharmD ${ }^{a, b}$, Susan Y. Kim, PharmDa,b, Judith C. Barker, \\ PhDc, Paul D. Blanc, $M D^{a, d}$
}

\author{
${ }^{a}$ California Poison Control System, San Francisco Division \\ bDepartment of Clinical Pharmacy, School of Pharmacy, University of California, San Francisco, CA \\ cDepartment of Anthropology, History \& Social Medicine, School of Medicine, University of California, San Francisco, CA \\ dDivision of Occupational and Environmental Medicine, Department of Medicine, School of Medicine, University of California, \\ San Francisco, CA
}

\begin{abstract}
Introduction: As part of a larger study assessing the covariates and outcomes of GHB use, we developed a telephone-survey instrument for hospitalized GHB exposed patients identified through poison control center surveillance and for self-identified GHB users recruited from the general public.

Methods: We used an iterative review process with an interdisciplinary team, including pharmacists, a physician, and a medical anthropologist. In designing the structured, telephone-survey instrument, we prioritized inclusion of validated, drug-specific, and generic questionnaire batteries or individual items related to GHB or to other drugs of abuse. Only one published survey instrument specific to GHB use was identified, which we extensively expanded and modified. We also developed a number of GHB-specific items new to this survey. Finally, we included items from the National Survey on Drug Use \& Health, CAGE questionnaire items on alcohol abuse, the SF-12 instrument, and selected National Health Interview items.

Results: The final questionnaire consisted of 272 content items, the majority of which required simple yes or no responses. The bulk of the items (74\%) were GHB-specific. The questionnaire was easily administered using computer-assisted telephone interview (CATI) software. A total of 131 interviews were administered with a mean administration time of $33 \pm 10$ minutes. The instrument can also be used in other interview formats.

Conclusion: Developing a successful questionnaire calls for a multidisciplinary and systematic process. Structured, telephone administered surveys are particularly suited to expand and explore the basic information obtained by poison centers for case management.
\end{abstract}

\section{INTRODUCTION}

Gamma hydroxybutyrate (GHB) emerged as a major new drug of abuse. Use as a body-building agent in the early 1990's led to wider recognition of the mood-altering effects of GHB. The abuse of GHB rapidly evolved, promoted through Internet marketing, as well as through the availability of precursor chemical agents that could either be converted to GHB ex vivo by way of chemical reactions prior to use or be metabolized in vivo to GHB after ingestion [1].

GHB's complex central nervous system manifestations are

Keywords: telephone-survey, methods, GHB, new drug of abuse

Notes: Source of Funding: NIDA GRANT RO1-DA 14935. Abstract Presented: European Association Poison Control and Clinical Toxicologists in Strasbourg, France June 2004; Short Title: GHB Survey Methods.

Corresponding Author: Jo E. Dyer, Pharm D, Clinical Professor of Pharmacy, California Poison Control System, San Francisco Division, UCSF Box 1369, San Francisco, CA 94143-1369. Email: jdyer@calpoison.org 
attributed to direct agonist effects at the $\mathrm{GABA}_{\mathrm{B}}$ receptor as well as action at another site specific to the agent (the so-called "GHB receptor") [2]. GHB's ability to produce memory impairment accompanied by a rapid loss of consciousness has been criminally exploited to facilitate sexual assault [3]. Another aspect of the drug that emerged with more widespread use is dependence and a withdrawal syndrome associated with escalating GHB administration [4]. In 2002, GHB was licensed as a restricted drug for cataplexy, thus providing another context for the drug's use and potential misuse [5].

All of the aforementioned characteristics make an understanding of GHB abuse important, but an understanding of abuse presents challenges to studying patterns of use and associated outcomes. Because GHB emerged as an entirely new drug of abuse, it was not specifically incorporated into the major relevant survey-based program, the National Survey on Drug Use and Health, NSDUH (formerly the National Household Survey on Drug Abuse). Poison control center case reporting is a major data source for drug overdose surveillance. As GHB abuse first started to emerge, poison control center case reporting was inconsistent in capturing GHB cases: this complicated the tracking of temporal trends, which now appear to be declining [6].

Another key limitation to epidemiological information on $\mathrm{GHB}$, especially for inferences made from surveillance sources, is that such data are collected in the context of acute medical events. Information on other aspects of GHB use and outcomes tends to be fragmentary or lacking altogether, such as data on user socioeconomic status, beliefs and attitudes (especially with regard to risk perception), co-use of other illicit substances, drugrelated psychosocial factors, and general health status. Such information is imperative for understanding the context of GHB abuse.

As part of the larger study of GHB effects, we wished to develop a structured survey instrument specific to GHB; the Factors in Overdose Research into GHB Effects (FORGE) study. The overall FORGE investigation includes analysis of surveillance data, controlled laboratory GHB exposures with formal pharmacokinetic and pharmacodynamic analysis, and a qualitative study component based on focus group interviews with users about their beliefs, perceptions, and practices. Consistent with the broad research agenda, our goal was to construct a survey instrument in order to collect information systematically on a wide range of factors relevant to GHB by recognizing its heterogeneous uses, wideranging effects, novel covariates of use, and the availability of multiple GHB congeners with potentially divergent dose response profiles (such as gamma butyrolactone or butanediol).

This investigation documents our approach to developing and implementing such a survey instrument, the FORGE Survey instrument. We believe that our experience might be generalized to the study of other emerging drugs of abuse that might appear in the future. Such substances are also likely to require a detailed and targeted collection of information to better understand and ultimately address adverse health effects associated with such outbreaks.

\section{METHODS}

\section{Overview}

In order to better assess correlates of use and risk factors for adverse outcomes associated with GHB, we developed a GHBspecific, telephone-administered, structured survey instrument. We designed this survey instrument to address:

- specific beliefs and practices related to GHB,

- the sources used in obtaining the drug and the circumstances surrounding its use,

- substances coingested with GHB whether licit or illicit,

- slang terms for GHB and its congeners,

- clinical symptoms related to acute and chronic GHB use,

- drug withdrawal-specific outcomes,

- subject demographics,

- socioeconomic status,

- psychosocial variables,

- and assessment of general physical and mental health status using standard quality of life measures.

In addition, we wished to include specific questions for interview subjects who experienced any GHB-related hospitalization or emergency department care. In particular, we wished to collect this information among a subset of the study cohort recruited into the study from case consultations obtained from the California Poison Control System (CPCS).

In this investigation, we analyze the process by which we constructed this survey instrument and the results of that effort, including the field implementation of the completed questionnaire. We systematically describe this process so that other researchers studying GHB can potentially apply our approach. We also believe that this methodological approach could be adapted in the study of other emerging issues in substance abuse; for example, any new toxic syndrome that might require targeted epidemiologic investigation utilizing a survey-research approach.

\section{Multidisciplinary Team in the Questionnaire Development Process}

In addressing the question of GHB use, we recognized that the first step would require a multidisciplinary approach. The rationale for such an approach was predicated on the realization that no single disciplinary perspective would sufficiently encompass the complex set of issues we wished to address in the content of the proposed survey. Consistent with this, an interdisciplinary survey instrument design committee was created to construct the questionnaire. The committee included members with expertise in the areas of internal medicine, toxicology, drug abuse and dependence, epidemiology, clinical pharmacy, and medical anthropology.

The committee met approximately every 4 weeks over a 6 month period from 2002 to 2003 to: 1) identify and review any preexisting validated survey instruments (Table 1) for content and relevancy to GHB and the overall goals of the investigation, 


\section{Table 1. Basis of Interview Content}

\begin{tabular}{|c|c|}
\hline $\begin{array}{l}\text { Sources of Questionnaire } \\
\text { Items [Reference Citation] }\end{array}$ & Total Items Contributed \\
\hline \multicolumn{2}{|l|}{ GHB-Specific Items } \\
\hline Miotto GHB Questionnaire [8] & 78 \\
\hline $\begin{array}{l}\text { National Survey on Drug Use and } \\
\text { Health items on dependence [9] }\end{array}$ & 31 \\
\hline FORGE study additions (this report) & 93 \\
\hline \multicolumn{2}{|l|}{ Other Substance Use Questionnaire Items } \\
\hline $\begin{array}{l}\text { National Survey on Drug Use and } \\
\text { Health items on drugs and tobacco [9] }\end{array}$ & 33 \\
\hline CAGE items on alcohol addiction [11] & 4 \\
\hline FORGE study additions (this report) & 5 \\
\hline \multicolumn{2}{|l|}{$\begin{array}{l}\text { Demographic, Mental and Physical } \\
\text { Health Items }\end{array}$} \\
\hline National Health Interview Survey [14] & 2 \\
\hline $\begin{array}{l}\text { National Survey on Drug Use and } \\
\text { Health demographic items [9] }\end{array}$ & 10 \\
\hline $\begin{array}{l}\text { 12-Item Short-Form Health } \\
\text { Survey - SF-12 [12] }\end{array}$ & 12 \\
\hline *FORGE study additions (this report) & 4 \\
\hline
\end{tabular}

2) develop specific wording for modified or new items incorporated into the questionnaire, 3) pre-test the new instrument, and 4) finalize the ultimate survey text and format. A key part of this process was the constant sharing among team members and documentation of prior and ongoing clinical experiences that addressed outcomes of GHB users, encounters with GHB users, and knowledge of their habits of use, beliefs, and practices. Broad familiarity with GHB proved essential for assessing adequacy and noting gaps in existing survey instruments as well as for discerning central issues for inclusion in the instrument under construction.

For each pre-existing questionnaire, we assessed whether each pre-existing questionnaire could be incorporated into the overall survey instrument in whole or in part through multiitem batteries or even selected individual items. We also evaluated whether an existing questionnaire or items within it, if not appropriate verbatim, is adaptable to our study goals by either word or format modification. When we could not identify any existing questionnaire components that addressed a study need, we used the committee process to develop and vet novel items for this purpose. The goal was to devise a questionnaire that would address relevant specific aspects of GHB use but to also systematically assess generic components of drug abuse and health.

\section{Team Goals and Process}

As a committee, we were readily able to determine several general guiding principles for our work. First, we set as a high priority a closed-ended question format, thus facilitating data collection in a uniform manner. Second, to better streamline data collection while still allowing detailed probing for applicable topics, we emphasized the use of skip patterns, preferably automated through the use of computer assisted telephone-administered (CATI) software. Skip patterns allow the respondent to "skip" to a destination further down the survey when a specific answer indicates an intervening section would not be applicable. Third, we understood that the questionnaire should be a maximum of 45 minutes in length. We viewed this time constraint as pivotal in extracting comprehensive information while still allowing willing participation and minimizing subject fatigue during the interview process. Fourth, we recognized that the questionnaire needed to use language appropriate for the likely mix of demographic populations surveyed, including slang synonyms for key drug usage and related behavior. Although we did translate the interview for administration into Spanish; ultimately, we did not employ this version because there were no monolingual Spanish interviewees. (We would like to note that translation into languages other than Spanish was not feasible).

Committee members were assigned specific topics to review in order to identify existing questionnaire items or to design new prototype items as needed. These would be circulated and discussed at subsequent committee meetings. Decisions for inclusion or exclusion were achieved by consensus. We did not seek out a systematic review by outside experts; for example, through "Delphi" iterative consensus building or a related process, but we did consult with identified experts external to our research team [7].

Our internal approach was iterative: we used a stepwise committee process of instrument refinement to address new issues, as they arose, in evolving the drafts that eventually became the final FORGE survey instrument. Thus, some components were initially included in draft form, but they were ultimately excluded as we achieved further refinement of the questionnaire through a pretesting and ongoing refinement phase. The final refinement phase of the committee process was necessary to maintain realistic time limitations that mandated an overall average length of administration of 45 minutes per subject.

\section{Developing the GHB-Specific Survey Content}

Creating GHB-specific content posed a key challenge. We began with systematic identification of any existing questionnaires specific to GHB. We consulted the National Library of Medicine's standard biomedical database, PubMed, using search terms such as gamma hydroxybutyrate or GHB and (survey or questionnaire or incidence or prevalence). Any identified publications were also reviewed for potentially relevant literature citations. Abstracts from the two major scientific meetings (the North American Congress of Clinical Toxicology and the European Association of 
Poisons Centers and Clinical Toxicologists) were also reviewed from 1998 to 2001 to identify the existence of applicable questionnaires not revealed through the PubMed search. In addition, we reviewed the data base of funded National Institute of Health research studies, CRISP, to identify other ongoing relevant research that has yet to generate publications. In addition, we directly contacted experts in the field of GHB to garner supplemental knowledge of any existing GHB-specific questionnaires.

Through this effort we identified only one existing GHBspecific questionnaire, that of Miotto and co-investigators [8]. Recognizing the unique nature of that instrument, we incorporated it into the FORGE survey instrument as a core portion of our GHBrelated items. The Miotto GHB questionnaire consists of a 45minute, semi-structured interview format administered by either a master's degree counselor or an addiction psychiatrist. It contains 175 individual response items and addresses demographic and psychiatric treatment history, as well as patterns of GHB use, its effects, sources, concomitant drug and alcohol use, and issues associated with the GHB dependence and withdrawal syndrome.

Our survey included nearly all of the GHB-specific items contained within the Miotto questionnaire. Questions regarding situational GHB use and frequency of GHB use were incorporated verbatim. Questions regarding concomitant drug use were incorporated with only slight modifications; for example, the question format remained virtually unchanged, although we modified the actual list of possible drug items that it contained. Specifically, we carried over 18 (out of 23) of the included substances. We removed 5 items because, based on Poison Control reports, there was not much likelihood of frequent coingestion with GHB (Quaaludes ${ }^{\circledR}$, barbiturates, morphine, nitrous, and Ambien ${ }^{\circledR}$ ). We also eliminated 3 generic categories for "other stimulants, other opiates, and other sedatives." Selected additions we added to the list included: Crack, Meth, Rohypnol®, Roofies, X, E, MDMA, DANS, Special K, ketamine, Coricidin ${ }^{\circledR}, \mathrm{DXM}$, and "shrooms." Slang terms were included in addition to generic drug names; for example, the common term "Ecstasy" as opposed to methylenedioxy-methamphetamine.

\section{Expanded Content}

To evaluate in greater depth factors that are possible predictors of adverse outcomes related to GHB, we expanded individual Miotto questionnaire items addressing the method, dosing, concentrations, interchange between GHB and its analogs, and route of administration. We also increased the list of sources from which the respondent might have obtained GHB. Examples of added response options included a personal trainer (given the importance of GHB use and dependence in body-building), medical doctor (given the FDA approval of prescription GHB, Xyrem ${ }^{\circledR}$ ), and Internet contact (given the emergence of the Internet as a key distribution route). We also added additional response options to an item querying the respondent's motivation for GHB use, including choices that seem consistent with dependence; for example, "to prevent GHB withdrawal symptoms" or "to prevent alcohol shakes or craving."

\section{Subjective Experience}

We modified a battery contained in the original Miotto questionnaire that elicited subjective effects experienced during and after GHB use. This battery was structured as a stem question followed by multiple response options that were not mutually exclusive. Because this list was quite lengthy and included a vocabulary that might not have been consistently comprehended, we removed 12 of the response options including the terms tranquility, giddiness, craziness, optimism, placidity, and well-being. Based on our clinical experience we added two new response options ("loss of memory or amnesia" and "involuntary muscle jerking") that are relevant and important in light of GHB's known adverse affects.

\section{Close-Ended Reformatting}

Finally, we restructured several open-ended questions in the Miotto questionnaire in order to conform to the closed-ended question format we had set as a priority for ease of administration and analysis. We preserved the core content of these items, which primarily concerned the situations of GHB use, GHB procurement sources, activities following GHB use, coingestants, and duration of the GHB "high." We also eliminated several open-ended questions that concerned activities the respondent would or would not do following GHB use and drugs that users would avoid coingesting with GHB.

\section{Novel GHB items}

Although we were able to meet our study priority of utilizing, to the extent possible, an existing GHB-specific questionnaire, critical issues remained for which the existing questionnaire was insufficient. This is not surprising because GHB has emerged as a relatively recent drug of abuse, has many unusual covariates, and has characteristics of use that have rapidly evolved.

Where possible, we addressed lacunae by simply expanding a set of response items, as noted above. Nevertheless, some issues remained for which the only feasible approach was to develop entirely new questionnaire items. For example, we devised 4 single-response questions specifically designed to assess the possible presence of aldehyde dehydrogenase (ADH) deficiency. We wished to address this topic because 1,4-butanediol is a GHB ana$\log$ /precursor whose metabolism is dependent on $\mathrm{ADH}$. We wanted the ability to assess whether subjects, with probable ADH deficiency, developed more significant clinical outcomes than the general population.

We also inserted a battery of 25 questions to assess the circumstances surrounding a respondent's GHB-related hospitalization. We did this because a key recruitment strategy of the FORGE study used CPCS case reporting of hospitalized, GHB-exposed patients to identify potential interview subjects. For those subjects recruited for interviews independent of CPCS reporting, these particular questions were skipped. In addition, a series of items further probed into other important issues. These items addressed eight subject areas: the type of product used (i.e., GHB as opposed to precursor drugs), the physical form of GHB 
ingested, any food intake prior to use, the temporal circumstances of GHB use (e.g., time of day, day of the week), sexual activity associated with use, driving a motor vehicle under the influence of GHB, other adverse reactions or physical injury secondary to GHB ingestion for which the subject did not seek medical care, and use of GHB via the FDA-approved prescription pharmaceutical product, Xyrem ${ }^{\circledR}$. To further enrich our understanding of the frequently changing vocabulary, we included an open-ended question to ascertain GHB slang names used by respondents.

\section{Dependence and Withdrawal}

We also realized the critical need for survey items that assessed GHB-dependence and GHB-withdrawal. These are important clinical entities that were only recently recognized after the expansion and frequency of GHB use during the latter 1990s. Although no validated GHB-specific items existed that addressed this phenomenon, we were aware that drug-dependence has been previously addressed using survey research methods.

Rather than relying on a "generic" dependence questionnaire, we favored adapting a substance-specific drug dependence battery and making it applicable to GHB. We did this by using the portion of the National Survey on Drug Use and Health (NSDUH), administered annually by the Substance Abuse Mental Health Services Administration (SAMHSA) instrument, that targets dependence for a series of drugs [9]. We adapted a version of the 2002 survey that asked a repeated battery of questions related to dependence for alcohol, marijuana or hashish, cocaine or crack, heroin, hallucinogens, inhalants, prescription pain relievers, tranquilizers, stimulants, and sedatives.

Because of the comprehensive nature of this battery and its easily understood questions in a well-tested format, we adapted this format by simply substituting the word GHB wherever the other drug of abuse appeared in the battery. When assessing GHB-specific withdrawal symptoms, we also used the same question format as in the NSDUH survey, but we devised a new list of eleven common, GHB-specific withdrawal symptoms pertaining to appetite, sleep, stimulation or sedation, hallucinations, and tachycardia.

To avoid repetition and increased length of survey with little added information, we considered, but ultimately did not include, questions drawn directly from the generic Addiction Severity Index [10]. We did review the Addiction Severity Index; however, it was done as a content cross-check with the overall adapted NSDUH battery. This review demonstrated that we had captured the appropriate subject matter relevant to dependence and withdrawal.

\section{Other Adapted Items}

In addition to the aspects of the questionnaire related to drug coingestion previously described, we also employed selected portions of the NSDUH survey to supplement content on other drug use and dependence as well as drug-related psychosocial issues. Portions of the NSDUH survey were used verbatim; other por- tions were adapted for GHB. Finally, we based our list of potential coingestants to expand the GHB-specific questionnaire (noted previously) on the content of the NSDUH survey. We also assessed tobacco use through incorporation of the battery used in the NSDUH. To screen for alcohol dependence among our subject population, we incorporated the four classic questions from the CAGE alcoholism assessment instrument into our questionnaire: Cut down on drinking, Annoyed at criticism about drinking, Guilty about drinking, Eye opener to steady nerves in the morning [11].

General mental and physical health status were assessed with the widely used twelve-item Short Form (SF-12) battery [12]. The SF-12 is concise, evaluates both physical and mental health status, and allows for comparison of general health-not only among individuals with other disease states but also among the general population. Finally, we collected information on subject demographics and socioeconomic status largely by incorporating the well standardized structure and content of selected National Health Interview Survey (NHIS) items (along with NSDUH items) $[13,14]$. Despite the potential sensitivity of the content areas of many, if not most of the items previously described, we realized some subjects might be reluctant to answer certain demographic questions (such as, occupation, income or the number of family members [suggesting children in the household]). And so, we placed many of these sensitive questions near the end of the survey instrument where failure to continue answering would not compromise the capture of as many GHB-related responses as possible.

\section{RESULTS}

\section{Survey Format}

The finalized questionnaire contained 272 content items. Of the total items in this FORGE Survey instrument, the subset of subjects who had been recruited through a CPCS referral as a hospital-treated case were asked 44 (16\%) of the items. Almost all of the questions were structured as "forced-choice" or "closedended" survey items. Of these, the majority elicited simple yes or no responses. For 67 response items, eleven response scale questions required respondents to choose from a range of possible options:

1. "How are you limited performing moderate activities? a lot, a little, not at all", or

2. "How would you rate your likelihood of using GHB to increase muscle mass?" asking for a response on a scale of " 0 " for Never and " 4 " for Always.

There were few wholly open-ended items included in the FORGE survey: there were four in total that assessed occupation type and industry (asked of employed subjects only), elicited any slang names used for GHB (all respondents were asked, "What name do you use for GHB?"), and an open-ended response option that followed a check-list of coingestants used with GHB that lead to a hospital evaluation (asked of the hospital subset only). 
In addition to these open-ended text field options, a limited number of open-ended queries of "other" followed an extensive list of response options in a "choose the best answer" format. For example, all subjects were asked the usual source and form of GHB taken; only the hospital group was asked to specify the place they last remembered before arriving at the hospital and the type of transportation used to reach the hospital.

Wherever possible, we employed skip patterns to take into account responses to items that would have led to redundancy in later items. For example, the questions "have you taken GHB together with Ecstasy" and "have you ever used Ecstasy," while not mutually exclusive, are hierarchical: a "yes" to the first question also answers the second. Thus the latter does not need to be asked following an affirmative response to the former. Skip patterns also took into account responses of hospital-treated subjects to avoid redundant questions.

\section{Survey Content}

The content sources of the final questionnaire are summarized in Table 1. GHB-specific content accounted for most of the items in the survey, although non-GHB substance use, abuse, and general content items related to demographics and health status were also important components. The final content of the 272-item questionnaire was $74 \%$ for GHB-specific items, $6 \%$ for other substance abuse, and $20 \%$ for demographics and general mental and physical health.

\section{Pilot Testing}

The final version of the questionnaire was piloted to assess completion time and ease of comprehension. Pilot testing was performed by direct face-to-face administration rather than by telephone. We used a convenience sample of 10 volunteers, including CPCS staff members and several high school and college students. These respondents easily answered all questions in a time period that ranged from 37 to 65 minutes. We did not identify any substantive need to change the final version of the questionnaire as a result of the pilot administration.

\section{Survey Administration}

We engaged an outside professional survey research firm to administer the structured telephone survey as finalized. The interview staff, having no specific prior content knowledge related to GHB, was educated about GHB by members of the study team. In addition to technical aspects of the questions, they were also trained to understand possible GHB-related responses that might arise. Altogether, approximately 4 hours of GHB-specific content training was provided.

Survey administration utilized CATI software to automate the key activities of the telephone interview and program the final survey instrument. During survey administration, skip patterns are automatically executed to avoid redundant questions, avoid the hospitalization specific questions for the non-hospitalized cohort, assure responses are within range, and limit missing data. Answers are entered directly into the computer database so an additional data entry step is eliminated. Data was exported in a format compatible with standard computer statistical analysis packages, such as SAS (Statistical Analysis System) or SPSS (Statistical Package for the Social Sciences).

Of 131 administered interviews, the mean administration time was $33 \pm 10$ minutes. The duration of interviews was not normally distributed; however, they ranged from 18 to 75 minutes (median time 31 minutes; $25^{\text {th }}-75^{\text {th }}$ percentiles, 26 to 38 minutes). Although the hospital-treated subjects were asked additional questions, the median administration time of the hospitaltreated subjects was slightly shorter (median=28 minutes) than among all other subjects (median=31 minutes).

\section{Survey Participation}

Volunteers provided written or verbal informed consent prior to enrollment. The Committee on Human Research at the University of California, San Francisco, approved the study; additionally, a Certificate of Confidentiality was obtained from the National Institutes of Health to further protect the identity of our participants. There were two principal recruitment venues.

One pool of subjects was identified through CPCS surveillance comprised of persons symptomatic with GHB or its analogs or precursors and who had received evaluation in a health care facility. While in hospital this cohort assented to provide home contact information to the study team. Study information and consent forms were mailed to potential study subjects, and subjects signed and returned the forms prior to actual participation.

A second, non-hospitalized group was recruited with Internet postings on Erowid and Project GHB websites, flyers in public places (such as bars, clubs, and laundromats), physician referrals, and from volunteers who have used GHB or its analogs or precursors. After an initial subject-initiated contact, the eligibility of subjects in the second group was confirmed after verbal consent through completion of a brief screening questionnaire. Subjects less than 16 years of age were excluded from the interview. Altogether, 146 consented subjects were contacted and interviewed, 14 subjects from the hospitalized group and 132 in the non-hospitalized group.

Of 146 subjects who were consented and referred for interview, 131 interviews were successfully completed (90\%). The 15 consented but uncompleted interviews were for the following reasons; 7 wrong telephone numbers, 3 non-working telephone numbers, 3 apparently valid phone numbers for which repeated left messages were not returned, and 2 refusals (only one of which cited confidentiality concerns).

\section{DISCUSSION}

Our investigation demonstrates how a careful, systematic, stepwise approach can be applied successfully to the development of a substance-specific questionnaire designed to access a complex set of use (or abuse) and outcome-related factors. Development of a survey instrument in this manner is resource intensive. The project described was carried out as part of a multi-component, Federally supported research effort (see acknowledgments for specifics of support); the questionnaire component involved a 
number of investigators whose time and effort was supported from this source in order to complete the questionnaire. Prior knowledge and experience with GHB practices, users, and outcomes was crucial to assessing the existing survey instruments and to redressing identified gaps during the construction of the FORGE survey instrument for GHB questionnaire.

Certain limitations are evident in our design, which intentionally restricted open-ended questions. This closed-ended format does not allow for qualitative data or capture of outlying response options. Although we did not pilot test the instrument on GHB users because we wished to garner this limited resource for the final survey administration, we did informally pilot the key sections with the study team itself. In addition, by relying on previously validated batteries, we minimized the need for extensive pilot testing. Moreover, the multi-component FORGE study design also included qualitative focus group discussions among light and heavy GHB users. Nonetheless, we acknowledge that these elements of pilot testing were limited and did not provide as extensive a component of pre-testing that is ideal prior to actual field administration. Such piloting might have included administration of the survey to the same subject face-to-face and then by telephone and also by pre-testing of dependent and nondependent GHB users. The former might have allowed us to better gauge the actual interview length, given that our face-to-face pilot testing under-estimated the actual saving of field-administration time achieved by use of the CATI, skip patterns, and avoidance of question redundancy along with telephone delivery. Our chief concern, however, was added length rather than shorter administration time. Pilot testing with dependent and non-dependent GHB users might have provided us with additional areas to explore in the questionnaire. In addition to the limitations of pilot testing, our survey methodology did not include a follow-up feedback-evaluation component post-administration that might have provided other insights missed in a onetime structured interview

Two other recent or ongoing GHB surveys are relevant to our study; although, questionnaire measures from these studies were not incorporated into our instrument. A study of GHB use and related adverse outcomes in Australia included a 30-45 minute structured interview that addressed GHB use patterns, perceptions, and adverse effects. It included a severity of dependence scale for GHB use as well as a drug-use-history that addressed lifetime and recent use (last 6 months) of drugs or alcohol [15]. The second relevant study of GHB, conducted at Minneapolis Medical Research Foundation, includes a battery of multiple GHB-specific items including a "craving scale", general drug use (as in the Australian study noted above), an addiction severity measure, and standardized depression and anxiety measures [16].

Poison control center-based epidemiological assessments of substance abuse have largely relied on retrospective data reviews. Investigations have either been limited to poison center records at a local or regional level or have used national data of the American Association of Poison Control Centers (AAPCC). In certain cases, poison center data have been compared to other data sources, for example DAWN reports or other surveys. Indeed, we recently reported the results of precisely such an investigation focusing on temporal trends in GHB incidence [6]. Such approaches, although extremely useful to address certain questions, are severely limited in their ability to capture many important aspects of drug use and the health outcomes that result from such practices. A recent Institute of Medicine Report, reviewing the function and role of poison control centers, emphasized some of these same strengths and weaknesses [17].

\section{CONCLUSION}

To improve the epidemiological research of poison control centers, rigorous survey research methods in general and questionnaire design are needed. Our experience in developing the FORGE instrument highlights some of the key elements in constructing and implementing a structured questionnaire approach to a prototypical research question.

The authors have no potential conflicts of interest to report.

\section{REFERENCES}

1. Dyer JE, Haller CA. Gamma Hydroxybutyrate and the Comatose Patient http://www.chestnet.org/education/pccu/ vol14/lesson21-22.index.html. Pulmonary and Critical Care Update Online 2001; 14 (lesson 22).

2. Snead OC, 3rd, Gibson KM. Gamma-Hydroxybutyric Acid. N Engl J Med 2005;352(26):2721-32.

3. ElSohly MA, Salamone SJ. Prevalence of Drugs used in cases of Alleged Sexual Assault. J Anal Toxicol 1999;23(3):141-6.

4. Dyer JE, Roth B, Hyma BA. Gamma-Hydroxybutyrate Withdrawal Syndrome. Ann Emerg Med 2001;37(2):147-53.

5. FDA. FDA Approves Xyrem for Cataplexy Attacks in Patients with Narcolepsy: U.S. Food and Drug Administration; 2002 July 17, 2002. Report No.: T02-31.

6. Anderson IB, Kim SY, Dyer JE, Burkhardt CB, Iknoian JC, Walsh MJ, et al. Trends in Gamma-hydroxybutyrate (GHB) and Related Drug Intoxication: 1999 to 2003. Ann Emerg Med

2006;47(2):177-83.

7. Dalkey N, Brown B, Cochran S. The Delphi Method, III: Use of Self Ratings to Improve Group Estimates. Santa Monica: The Rand Corporation; 1969 November 1969. Report No.: RM-6115-PR.

8. Miotto K, Darakjian J, Basch J, Murray S, Zogg J, Rawson R. Gamma-Hydroxybutyric Acid: Patterns of use, effects and withdrawal. Am J Addict 2001;10(3):232-41.

9. NSDUH. Overview of findings from the 2002 National Survey on Drug Use and Health. Rockville, MD: Substance Abuse and Mental Health Services Administration; 2003 September 2003. Report No.: OAS, NHSDA Series H-21, DHHS Pub \# SMA 03-3774.

10. McLellan AT, Kushner H, Metzger D, Peters R, Smith I, 
Grissom G, et al. The Fifth Edition of the Addiction Severity Index. J Subst Abuse Treat 1992;9(3):199-213.

11. Mayfield D, McLeod G, Hall P. The CAGE Questionnaire: Validation of a new alcoholism screening instrument. Am J Psychiatry 1974;131(10):1121-3.

12. Ware J, Kosinski M, Keller SD. A 12-Item Short-Form Health Survey: Construction of scales and preliminary tests of reliability and validity. Med Care

1996;34(3):220-33.

13. Adult Health Practices in the United States and Canada. Washington D.C.: National Center for Health Statistics; 1988.
14. Kovar M, Poe G. National Health Interview Survey Design, 1975-1984 and Procedures, 1975-1983; 1985.

15. Degenhardt L, Darke S, Dillon P. GHB use among Australians: characteristics, use patterns and associated harm. Drug Alcohol Depend. 2002 Jun 1;67(1):89-94.

16. Zvosec, DL, Smith SW. Preliminary Findings from the GHB addiction study, MMRF. (Abstract) Presented: International GHB \& Chemical Drug Conference September 25-27, 2004, Las Vegas NV.

17. Press NA. Committee on Poison Prevention and Control, Board on Health Promotion and Disease Prevention. Forging a poison prevention and control system. Washington D.C: Institute of Medicine; 2004. 